\title{
IMMUNOCHEMISTRY
}

\section{Quantum dots go with the flow}

Adding quantum dots to a repertoire of conventional fluorochromes allows researchers to extend the power of fluorescence immunophenotyping.

Most often associated with stunning micrographs, quantum dots have been widely used in the imaging field because of the sensitivity they afford. But Mario Roederer at the US National Institutes of Health Vaccine Research Center, and his colleagues, adopted these fluorochromes to improve the ability to resolve subsets of immune cells.

The complexity of the immune system translates into a combination of different proteins on the surface of each cell type, and these markers can be identified by fluorescent antibody detection and subsequent flow cytometry analysis_-a process known as immunophenotyping.

"What we find is that we can't adequately describe the functionalities of the immune compartment by just making a single measurement like 'Do they make gamma

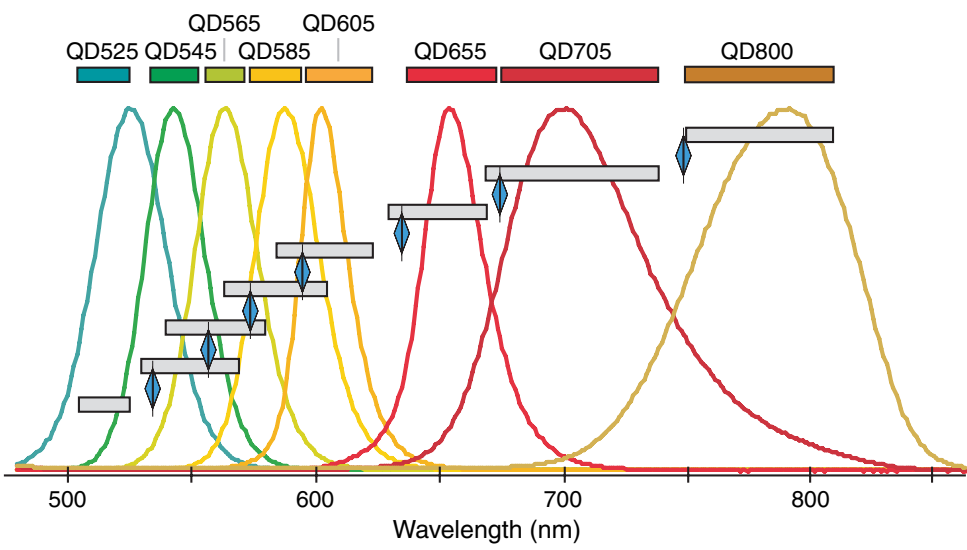

Figure 1 | Emission spectra of various quantum dots. Blue diamonds mark the wavelengths for longpass dichroic filters, and band-pass filters are shown as gray bars. Image reprinted with permission from Nature Medicine.

interferon?' or 'Do they divide or not?', but it's the combination of all these measurements that will start resolving interesting subsets," says Roederer. With organic fluorochromes, Roederer and col- leagues had been able to achieve 12-color flow cytometry. Addition of quantum dots, with their limited spectral overlap (Fig. 1), allowed them to resolve 18 colors to date.

\section{RNA INTERFERENCE}

\section{IN PRAISE OF MANUAL HIGH-THROUGHPUT SCREENING}

RNA interference (RNAi) and automated high-throughput screening is a promising combination. But the first systematic large-scale mapping of genetic interactions in an animal shows that manual methods still have advantages over sophisticated automated screens.

Despite the fact that many diseases likely result from mutations in multiple genes, there is very little in vivo data on the genetic interactions that underlie these phenotypes in complex organisms. This is largely due to the difficulty in genetically manipulating animals compared to yeast or bacteria. RNAi, however, is making large-scale genetic screens in animals feasible.

Because RNAi can reduce the expression of a specific gene, it can be used to generate pseudo-knockout animals with the same phenotype as a genetic knockout even though they still possess the gene of interest. The trick is to be able to efficiently deliver small interfering RNA to the animal and to quickly screen for phenotypes-two criteria that Caenorhabditis elegans fulfills beautifully.

While in Julie Ahringer's laboratory, Andrew Fraser became an expert at using RNAi in $C$. elegans screens. In work just published he has used these techniques to systematically test $\sim 65,000$ pairs of genes for genetic interactions. You might expect such a large scale experiment to require the development of a complex and sophisticated automated approach, but you would be wrong.

In fact, the major methodological change was the use of a 96well liquid format for feeding small interfering RNA-containing bacteria to $C$. elegans instead of agar plates. And their readout? Well, they looked at the worms. As Fraser puts it, "I always tend to go low-tech before I go high-tech. I want to make sure that if we do these screens it gives us biologically informative data before I build a very clever way to do it."

In vivo screens are much more difficult to automate than screens in cell lines. "The problem in vivo is that until you've had a look at the data you're not quite sure what you should be looking for. You can miss lots of the weird phenotypes because you didn't tell your machine how to count them-teaching a machine to find the unexpected is hard. Those are things that you rarely miss by eye," says Fraser.

Not only can manual examination spot phenotypes that might be missed by automated phenotyping, bizarrely it can also be faster because the human eye spots wild-type phenotypes with barely a glance while a computer would have to acquire an image and analyze it. Fraser remarks, "The manual method is really amazingly quick for that, and there is nothing we have automated that even gets close." 
Selection of the fluorophore panel for a given experiment, however, remains a trial-and-error process. Based on their extensive experience working with the fluorochromes, Roederer and colleagues match the brightest reagents with less-abundant markers, keeping in mind that spectral overlap reduces sensitivity. Yet to develop a large panel of fluorophores, they test several different colors of each of the antibodies they want to use. "We start by mixing several different combinations of six or eight antibodies and see how that performs, and then we start layering in additional reagents and continually revising the panel," explains Roederer.

In new work reported in Nature Medicine, they used quantum dot-conjugated antibodies in combination with organic fluorochrome-conjugated antibodies to assay the phenotype of antigenspecific $\mathrm{T}$ cells. In this 17 -color analysis, they were able to identify the expected markers, but also found new phenotypic differences between the populations.

As in imaging, the addition of quantum dots to the palette of fluorescent tags affords greater sensitivity. As Roederer puts it, "Historically, we've been limited to three or four different tags, but now we are working our way to where we have several dozen, and that gives us just much more flexibility in designing and implementing experiments where we want to detect fluorescent tags."

\section{Irene Kaganman}

\section{RESEARCH PAPERS}

Chattopadhyay, P.K. et al. Quantum dot semiconductor nanocrystals for immunophenotyping by polychromatic flow cytometry. Nat. Med. 12, 972-977 (2006).

This low-tech method allowed Fraser and colleagues to identify 349 interactions out of $\sim 65,000$ tested, which means that pairwise combinations of mutations are many times more likely to result in nonviable phenotypes than single mutations. And this is only looking at the highest-confidence hits.

To try to identify weaker interactions, Fraser is now building an automated image analysis system. He explains, "Now at least we have test sets so we know how well the screen works, what it gives us, and that it is worth doing." Fraser is quick to add, however, that even if they get the automated method working well they will likely do a very rapid manual screen as a first pass and then do all the repeats in a quantitative way using the automated screening.

So before you embark on your next high-throughput screening experiment, it might be worth considering whether it might be good to do an old-fashioned manual screen before investing time and effort in something that does not work out quite as planned.

\section{Daniel Evanko}

\section{RESEARCH PAPERS}

Lehner, B. et al. Systematic mapping of genetic interactions in Caenorhabditis elegans identifies common modifiers of diverse signaling pathways. Nat. Genetics 38, 896-903 (2006).

\section{NEWS IN BRIEF}

\section{PROTEIN BIOCHEMISTRY}

\section{Experimental generation of electrostatic potential maps}

Red, white and blue electrostatic potential maps are often computationally generated for protein structures to assess the roles of electrostatics in various protein functions. Suydam et al. now demonstrate that these maps can be experimentally generated for the active site of human aldose reductase, by mapping the effects of the local electrical field on a nitrilebased inhibitor using vibrational spectroscopy.

Suydam, I.T. et al. Science 313, 200-204 (2006).

\section{MOLECULAR LIBRARIES}

\section{Improving the display of proteins on phage}

Phage display is a powerful tool for the high-throughput screening of proteins for desired properties. Unfortunately, many proteins display poorly on phage despite attempts to address the problem. Steiner et al. have vastly improved the range of proteins amenable to phage display by using signal sequences that harness the signal-recognition particle translocation pathway rather than the traditional Sec-dependent translocation pathway. Steiner, D. et al. Nat. Biotech. 24, 823-831 (2006).

\section{GENE TRANSFER}

\section{On-target gene delivery with lentiviral vectors}

Lentiviral vectors have shown great potential as in vivo gene delivery vehicles, but assuring on-target delivery is very difficult. Yang et al. now show that efficient delivery of the vector to specific cell types is possible by separating the functions of target recognition and membrane fusion into two unique moieties on the lentiviral surface-an antibody and a $\mathrm{pH}$-responsive fusogenic envelope glycoprotein. Yang, L. et al. Proc. Natl. Acad. Sci. USA 103, 11479-11484 (2006).

\section{CHEMICAL TOOLS}

\section{Fluorescent dyes for live-cell imaging of RNA}

By using combinatorial organic synthesis to generate a large fluorescent styryl dye library, Li et al. discovered three RNAselective probes that can be used for the live-cell imaging of RNA distribution within the nucleus. These three dyes exhibited higher selectivity, photostability and lower cytotoxicity compared with a commercially available alternative. Li, Q. et al. Chem. Biol. 13, 615-623 (2006).

\section{SPECTROSCOPY}

\section{Probing membrane protein orientation with EPR spectroscopy}

Inbaraj et al. report a new technique for determining integral membrane protein topology in bicelles by electron paramagnetic resonance (EPR) spectroscopy. Using the nicotinic acetylcholine receptor as an example, they show that the incorporation of a nitroxide spin probe facilitates measurement of hyperfine splittings, which are used to accurately calculate the helical tilt of the transmembrane domain.

Inbaraj, J.J. et al. J. Am. Chem. Soc. 128, 9549-9554 (2006). 